\title{
Cognitive Improvement and Brain Changes after Real-Time Functional MRI Neurofeedback Training in Healthy Elderly and Prodromal Alzheimer's Disease
}

\author{
Christian Hohenfeld 1,2,3, Nils Nellessen ${ }^{1,2,3}$, Imis Dogan ${ }^{1,2,3}$, Hanna Kuhn ${ }^{1,2,3}$, \\ Christine Müller ${ }^{1,2,3}$, Federica Papa ${ }^{1,2,3}$, Simon Ketteler ${ }^{1,2,3}$, Rainer Goebel ${ }^{4,5,6}$, \\ Armin Heinecke ${ }^{6}$, N. Jon Shah ${ }^{1,2,3}$, Jörg B. Schulz ${ }^{1,3}$, Martina Reske ${ }^{2,3}$ and Kathrin Reetz ${ }^{1,2,3 *}$ \\ 'Department of Neurology, RWTH Aachen University, Aachen, Germany, ${ }^{2}$ Institute of Neuroscience and Medicine (INM-4, 6), \\ Research Centre Jülich GmbH, Jülich, Germany, ${ }^{3} J A R A-B R A I N$ Institute Molecular Neuroscience and Neuroimaging, \\ Forschungszentrum Jülich GmbH and RWTH Aachen University, Aachen, Germany, ${ }^{4}$ Department of Cognitive Neuroscience, \\ Maastricht University, Maastricht, Netherlands, ${ }^{5}$ Maastricht Brain Imaging Centre, Maastricht University, Maastricht, \\ Netherlands, ${ }^{6}$ Brain Innovation, Maastricht, Netherlands
}

OPEN ACCESS

Edited by: Itamar Ronen,

Leiden University, Netherlands

Reviewed by: Hidenao Fukuyama, Kyoto University, Japan Konstantinos Kalafatakis,

University of Bristol, United Kingdom

*Correspondence: Kathrin Reetz kreetz@ukaachen.de

Specialty section: This article was submitted to Applied Neuroimaging,

a section of the journal

Frontiers in Neurology

Received: 31 May 2017 Accepted: 19 July 2017 Published: 09 August 2017

Citation: Hohenfeld C, Nellessen N, Dogan I, Kuhn H, Müller C, Papa F, Ketteler S, Goebel R, Heinecke A, Shah NJ, Schulz JB, Reske $M$ and Reetz K (2017) Cognitive Improvement and Brain Changes after Real-Time Functional MRI Neurofeedback Training in Healthy Elderly and Prodromal Alzheimer's Disease.

Front. Neurol. 8:384. doi: 10.3389/fneur.2017.00384
Background: Cognitive decline is characteristic for Alzheimer's disease (AD) and also for healthy ageing. As a proof-of-concept study, we examined whether this decline can be counteracted using real-time fMRI neurofeedback training. Visuospatial memory and the parahippocampal gyrus (PHG) were targeted.

Methods: Sixteen healthy elderly subjects (mean age 63.5 years, SD $=6.663$ ) and 10 patients with prodromal $A D$ (mean age 66.2 years, $S D=8.930$ ) completed the experiment. Four additional healthy subjects formed a sham-feedback condition to validate the paradigm. The protocol spanned five examination days (T1-T5). T1 contained a neuropsychological pre-test, the encoding of a real-world footpath, and an anatomical MRI scan of the brain. T2-T4 included the fMRI neurofeedback training paradigm, in which subjects learned to enhance activation of the left PHG while recalling the path encoded on T1. At T5, the neuropsychological post-test and another anatomical MRI brain scan were performed. The neuropsychological battery included the Montreal Cognitive Assessment (MoCA); the Visual and Verbal Memory Test (VM); subtests of the Wechsler Memory Scale (WMS); the Visual Patterns Test; and Trail Making Tests (TMT) A and B.

Results: Healthy elderly and patients with prodromal AD showed improved visuospatial memory performance after neurofeedback training. Healthy subjects also performed better in a working-memory task (WMS backward digit-span) and in the MoCA. Both groups were able to elicit parahippocampal activation during training, but no significant changes in brain activation were found over the course of the training. However, Granger-causality-analysis revealed changes in cerebral connectivity over the course of the training, involving the parahippocampus and identifying the precuneus as main driver of activation in both groups. Voxel-based morphometry showed increases in grey matter volumes in the precuneus and frontal cortex. Neither cognitive enhancements, nor parahippocampal activation were found in the control group undergoing sham-feedback. 
Conclusion: These findings suggest that cognitive decline, either related to prodromal AD or healthy ageing, could be counteracted using fMRI-based neurofeedback. Future research needs to determine the potential of this method as a treatment tool.

Keywords: cognitive training, mental imagery, parahippocampus, plasticity, visuospatial memory, neurofeedback, real-time fMRI

\section{INTRODUCTION}

Alzheimer's disease (AD) is an age-associated neurodegenerative disease. It is the most common type of dementia, but no satisfactory treatment for it has been established yet, posing a major challenge for an ageing society $(1,2)$.

Alzheimer's disease is characterised by decline in cognitive function, especially in episodic memory. A domain affected the earliest and severest is visuospatial memory (3-5). Visuospatial memory is associated with the parahippocampal gyrus (PHG) (6), which has been shown to be affected by loss of grey matter (GM) in $\mathrm{AD}$, supposedly related to the visuospatial memory impairment $(7,8)$. Findings of GM loss are in line with neuropathological changes in early AD (9).

Patterns in cognitive decline, similar to $\mathrm{AD}$ can be observed in healthy ageing. While much less severe, healthy ageing is also characterised by cognitive decline (10). Again, visuospatial memory is one of the domains affected the earliest and the most pronounced (11).

An approach to counteract cognitive decline in healthy individuals and patients is cognitive training of specific domains. Successful training regimes have been reported in the literature (12-14). Besides cognitive effects, it was also reported that cognitive training can induce changes in brain structure in the healthy elderly (15) and patients of subjective cognitive impairment (16). Overall, evidence from cognitive training suggests cognitive decline could be at least partially reversible.

During recent years, neurofeedback using real-time functional magnetic resonance imaging (rtfMRI) has become an established method (17). The general idea of neurofeedback is that subjects are provided with information about the state of the brain activation. In the context of fMRI, the activation of one or more regions is usually visualised. Subjects then try to develop strategies to modulate the brain activation and assess the success of these strategies using the provided feedback (18). With the technology available today it has become possible to analyse fMRI data as it is acquired, but general limitations regarding the temporal resolution of fMRI data still apply. Despite its relative novelty, rtfMRI neurofeedback training has already been used in many clinical and non-clinical applications. Research demonstrated improvement of symptoms, e.g., in Parkinson's disease $(19,20)$, tinnitus (21), and major depression (22). Two studies on healthy subjects reported improvement of workingmemory performance after rtfMRI neurofeedback training of the dorsolateral prefrontal cortex $(23,24)$. In addition to these findings, a study employing electroencephalography (EEG)based neurofeedback training combined with diffusion tensor imaging of the brain, showed that structural brain changes could be induced (25).
Due to the results reported in cognitive training studies regarding cognitive decline and due to the success of neurofeedback applications in many diseases, the present study applied rtfMRI neurofeedback training to prodromal $\mathrm{AD}$ (pAD) patients (26) and the healthy elderly. Due to their crucial roles in $\mathrm{AD}$ and healthy ageing, we chose visuospatial memory and the PHG as training targets. Subjects would actively recall visuospatial memories while simultaneously trying to increase PHG activation.

We hypothesised that the training paradigm would enhance visuospatial memory performance and also induce changes in brain structure and function in regions related to visuospatial memory, mental imagery, and cognitive control.

\section{MATERIALS AND METHODS}

\section{Participants}

30 subjects completed the study. Subjects were divided into three groups: healthy elderly experimental group (HC, $n=16)$, healthy elderly sham-feedback group serving as a control group to verify whether feedback of PHG activation is required ( $\mathrm{SH}, n=4)$ and patients of $\mathrm{pAD}(\mathrm{PA}, n=10)$. Inclusion criteria were age between 50 and 80 years; right-handedness as determined by a simplified version of the Edinburgh Handedness Inventory (27); native language German; and the ability to provide written informed consent. Exclusion criteria included a history of neurologic or psychiatric disorders (except for pAD in group PA); concurrent use of psychotropic medication; MRI contraindications; familiarity with the Research Centre Jülich campus (see below); colour-blindness.

Healthy subjects were recruited in the RWTH Aachen university hospital and its vicinity. Patients of $\mathrm{pAD}$ were referred to the study from the memory clinic of the neurological department of RWTH Aachen University after being diagnosed according to the relevant criteria (26). AD diagnoses were based on a clinical and standardised neuropsychological test battery (CERAD) (28), MRI and cerebrospinal fluid-related neurodegeneration markers: amyloid $\beta_{1-42}$, amyloid $\beta_{1-40}$, amyloid $\beta_{1-42} / \beta_{1-40}$ ratio, total tau, and phospho-tau.

After full introduction of the study, all subjects provided written informed consent for participation. The local institutional review board at RWTH Aachen University approved the experiment and its procedures in accordance with the declaration of Helsinki (29).

\section{Study Design and Protocol}

The study protocol consisted of five examination days (T1-T5), with a time interval of 2-7 days in between. The interval was 
chosen based on previous studies, but also mandated by constraints in MRI scanner availability. Due to scheduling problems, this interval was exceeded in some cases: in group HC once in five subjects and twice in two subjects (mean $=9.904, \mathrm{SD}=18.392$, median $=3$ ); in group PA once in six subjects (mean $=7.05$, $\mathrm{SD}=9.438$, median $=5$ ); in group $\mathrm{SH}$ once in one subject $($ mean $=4.313, \mathrm{SD}=2.358$, median $=5)$.

A quasi-experimental design consisting of pre-test (T1), intervention (T2-T4), and post-test (T5) was employed. At T1, a neurological and neuropsychological examination, the visuo-spatial memory task of encoding a real-world footpath, and an anatomical MRI scan of the brain were performed. At T2-T4, rtfMRI neurofeedback training took place, in which the subjects were asked to recall the footpath encoded in the visuospatial memory task (see below for details). This was followed by a neuropsychological examination and another anatomical scan of the brain at T5. All examinations were carried out at the Research Centre Jülich, Germany. The procedures are summarised in Figure 1.

\section{Neurological, Psychopathological, and Neuropsychological Examination}

Trained professionals rated the neurological health and cognitive abilities of subjects. Neurological symptoms with a focus on memory-related problems were queried in a semi-structured interview and all participants received a basic neurological examination. Subjects were assessed using the SKID I (30) screening questions, a structured interview for diagnosis of psychiatric disorders from DSM-IV axis I (31). Also, subjects filled in the Beck Depression Inventory II (BDI-II) (32) to quantify self-experienced symptoms of depression.

Tests of the neuropsychological battery were selected based on the following criteria: (1) visuospatial memory should be represented; (2) a broad range of cognitive abilities should be metered

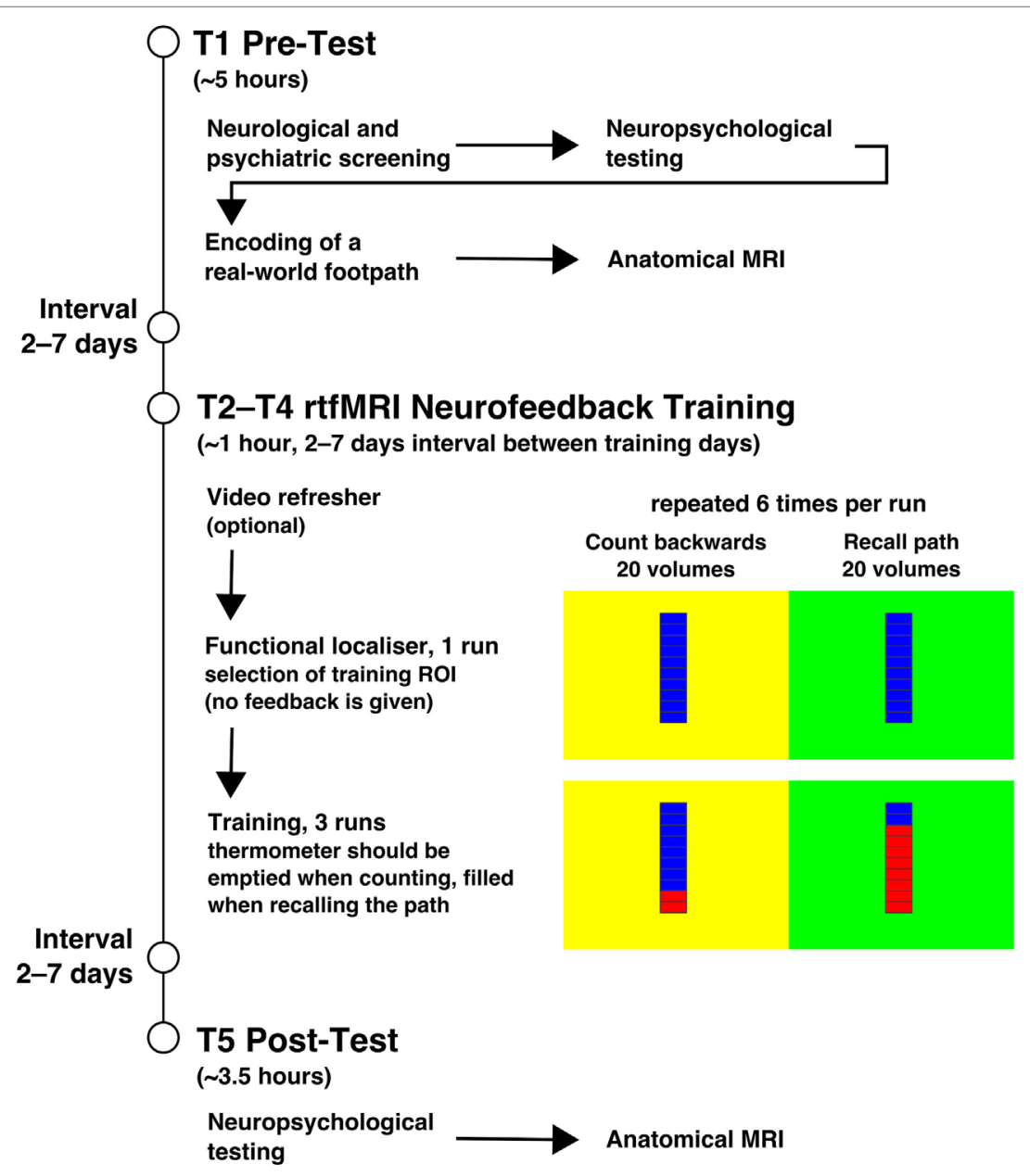

FIGURE 1 | Procedures. The figure summarises the procedures of the experiment. The experiment started with the pre-test on T1 and the illustrated procedure. T2-T4 included the neurofeedback training, which is displayed with more detail. Each training session started with the functional localiser in which the feedback stimulus was shown, but no feedback was given. It was used to select a region of interest (ROI) in the left parahippocampus as feedback source for the training. Feedback was shown during the three subsequent training runs. The background colour of the stimulus indicated the task with it being counting backwards at yellow and recalling the path from $\mathrm{T} 1$ at green. After the three training days, at T5 the post-test took place. The interval between all training days was usually 2-7 days. 
so potential generalisation effects could be observed; (3) due to repeated testing availability of parallel test versions implementing the same task with different contents was important. The test battery comprised of the following tests: the Visual and Verbal Memory Test (VVM) (33) on visuospatial and verbal memory, with immediate and delayed recall conditions; the subtests on visual and verbal memory both with immediate and delayed recall, as well as the forward and backward digit-span tasks of the revised Wechsler Memory Scale (34) to assess short-term memory and working-memory capabilities. For a short assessment of overall cognitive performance, the Montreal Cognitive Assessment (MoCA) $(35,36)$ was used. From the CERAD Plus (28), Trail Making Tests A and B (TMT-A and TMT-B), which test cognitive processing speed (TMT-A and -B) and task switching ability (TMT-B) were drawn on. The Visual Patterns Test (VPT) (37), which measures the capacity of visual working memory was further included into the test battery. Only at T1, the Multiple Choice Word Test (MWT-B) (38) as an estimate of premorbid intelligence was administered.

\section{Visuospatial Memory Task: Memorising a Real-World Footpath}

At T1, subjects encoded one out of three predefined real-world footpaths ("central," "west," "north") on the campus of the Research Centre Jülich. Paths were randomly assigned to subjects. From group HC, five subjects learned path central, five west, and six north. From group PA, three subjects learned path central, six west, and one north. From group SH, two subjects learned path central, another two subjects learned path west.

The instruction was to memorise as many details of the path as possible while the examiner guided the subject along the path. A stop was made at 10 waypoints (prominent landmarks, usually buildings with unique features). At each stop, subjects were informed about the name of the waypoint and they were asked to specifically memorise the waypoint. As soon as subjects indicated that they were certain that they had memorised the waypoint, the tour was continued.

Paths were between 1.5 and $2 \mathrm{~km}$ long, and a single tour was usually completed within $30 \mathrm{~min}$. Paths were mostly exclusive with only few crossing sections. At the beginning of each of the following sessions, subjects could watch a 3-min fast-forward video of the learned path to assure sufficient memory.

\section{Brain Imaging}

Brain imaging was performed on a Siemens (Erlangen, Germany) MAGNETOM Trio whole-body 3T MR scanner using standard gradients and a 32-channel phased-array head coil for signal reception; the body coil was used for radiofrequency transmission. Participants were positioned head-first supine. Foam padding was used within the head coil to limit movement. An MR-compatible mirror system was attached to the coil so that subjects could look out to the back of the scanner where an MR-compatible LED screen was mounted.

\section{Imaging Parameters}

On all examination days, high-resolution anatomical scans using a magnetisation-prepared rapid gradient echo (MP-RAGE) sequence were acquired $(\mathrm{TR}=2,250 \mathrm{~ms}$, $\mathrm{TE}=3.03 \mathrm{~ms}$, flip angle $=9^{\circ}$, slice thickness $=1 \mathrm{~mm}, 256 \times 256$ in-plane matrix, acquisition time: $5 \mathrm{~min} 14 \mathrm{~s}$ ). For rtfMRI neurofeedback and functional localiser runs, an echo planar imaging sequence was used $\left(\mathrm{TR}=2,000 \mathrm{~ms}\right.$, TE $=62 \mathrm{~ms}$, flip angle $=79^{\circ}$, field-ofview $=192 \times 192,64 \times 64$ in-plane matrix, 32 slices with $3 \mathrm{~mm}$ thickness and an inter-slice-gap of $1.2 \mathrm{~mm}, 240$ whole-brain volumes, acquisition time: $8 \mathrm{~min}$ ).

\section{Neurofeedback Training: Setup and Implementation}

On T2-T4, the neurofeedback training was performed. Following the anatomical scan, a functional localiser run and three neurofeedback training runs were carried out. MRI data were transferred by network from the scanner console to an additional computer for real-time analysis. Images were analysed using Turbo-BrainVoyager (Brain Innovation, Maastricht, The Netherlands) and the analysis result (a relative numerical value of activation) was written to disk and read by a Python (https:// www.python.org/) script within PsychoPy (39) to generate the visual feedback stimulus.

The functional localiser of each training day enabled the experimenter to select a region of interest (ROI) for the subsequent feedback runs using an overlay of functional results on anatomical data. For groups $\mathrm{HC}$ and PA, a ROI within the left PHG was selected as training target. For group $\mathrm{SH}$, a region from the left primary somatosensory cortex was chosen. The ROI had a size of 500-700 voxels (vx) and was selected at a statistical threshold of $t \geq 3$ in $\mathrm{HC}$ and PA; for $\mathrm{SH}$ the activation threshold was lowered as far as required to select a ROI in the desired region. ROIs from two subjects are visualised in Figure 2. Brain activation derived from the selected ROI was visualised as a thermometer bar during neurofeedback training runs (Figure 1). The task during the functional localiser was the same as in the subsequent training runs (see below), but no visual feedback was provided.

During training runs, baseline phases and upregulation phases (six each) constantly alternated, lasting 20 whole-brain acquisitions ( $40 \mathrm{~s}$ ) each. Phases were indicated by the background colour of the visual feedback stimulus, with yellow indicating baseline phases and green indicating upregulation phases.

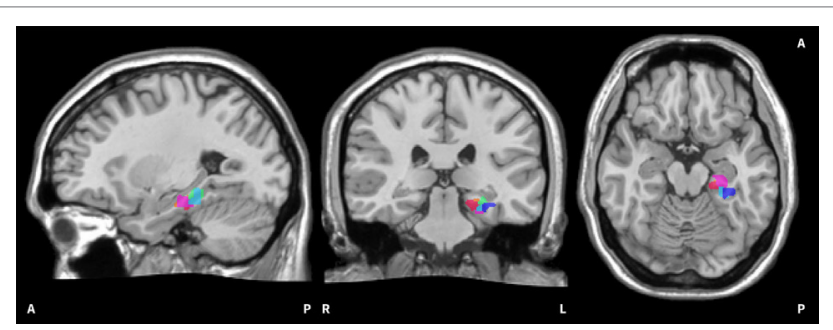

FIGURE 2 | Visualisation of on-line regions of interest (ROIs). The image shows all on-line ROls from two exemplary subjects. The red/pink hues belong to the first subject, the blue/green hues to the second subject. It can be seen that the selected ROIs in the left parahippocampus are scattered to a certain extent. The ROls are superimposed onto the Colin 27 average brain. Copyright@ 1993-2009 Louis Collins, McConnell Brain Imaging Centre, Montreal Neurological Institute, McGill University. 
Upregulation phases required subjects to actively remember and imagine the path they had learned at T1 to try enhancing brain activation visualised in the thermometer bar. Baseline phases required subjects to slowly count backwards from 100 to bring down the thermometer bar. The baseline condition was designed to be very different from the spatial navigation task in the upregulation phases and thus to elicit different patterns of brain activation. Each rtfMRI neurofeedback session lasted about $40-45$ min.

\section{Debriefing after rtfMRI Runs}

On each training day, participants were debriefed after the functional localiser and after each of the three feedback runs to ensure that all subjects followed the given instructions. After the functional localiser, subjects were asked whether they had been able to recall the learned path well (yes or no). Further, subjects were asked what they had done when the background of the thermometer was yellow and green, respectively. Last, subjects were asked whether they felt that they were able to influence the thermometer (yes/partially/no).

\section{Statistical Analysis \\ Behavioural Analysis}

Neuropsychological test scores were transformed into percent ranks according to normative data provided with the tests' manuals, stratified by age, education and/or gender (WMS: age; VVM and VPT: age and education; TMT-A/B: age, education, gender). For the MoCA, raw scores were used and for the MWT-B an estimated premorbid intelligence quotient was generated.

Data were analysed using R (40). For each neuropsychological test a linear mixed model was calculated, using examination time point as within-subject predictor and if the normative data for the specific test did not already correct for it gender and/or age as additional predictors. To assess model fit $\Omega_{0}^{2}$ is reported (41). Furthermore age, BDI-II score, and MWT-B score were tested for differences between groups using an ANOVA. The significance threshold was set to $\alpha=0.05$.

If a data point from a subject in a neuropsychological test was missing, that data point was excluded from the respective analysis. This was true for two subjects from the group HC, with data missing due to technical problems for the WMS verbal memory task: in one case on $\mathrm{T} 1$ and $\mathrm{T} 5$, respectively.

\section{fMRI Analysis}

The fMRI data gathered during training sessions was analysed using the software BrainVoyager QX, the NeuroElf toolbox (http://neuroelf.net/) for Matlab (The MathWorks, Natick, MA, USA) and R to extract and analyse time course data.

Functional data were pre-processed using Brain Voyager QX, employing slice scan time correction, three-dimensional motion correction and high-pass filtering using Fourier analysis (cutoff of two sine and cosine cycles over the course of the data). Additionally, spatial smoothing with a Gaussian kernel of $6 \mathrm{~mm}$ full-width at half-maximum (FWHM) was conducted. Anatomical data were skull stripped, intensity inhomogeneity corrected, and transformed to Talairach space. Functional and anatomical data were then co-registered and combined into a single dataset.

To assess brain activation, a general linear model (GLM) with a single predictor of upregulation and movement parameters as covariates was computed with Brain Voyager QX for each neurofeedback run within a group. Brain activation was assessed at uncorrected $p<0.001$ and at a threshold of Bonferroni corrected $p<0.05$. Minimum cluster size was determined using Alphasim (42) within Neuroelf. To identify brain regions active during all neurofeedback training runs, data were then averaged across all nine neurofeedback runs and a table of active clusters was generated.

Using R, the course of activation was extracted from the preprocessed fMRI data for a cluster of the left PHG as determined by the GLM. Data were averaged over the entire cluster per run and subject in a first step and then the percent signal change (PSC) was calculated. Here, the second half of each baseline phase was used as reference for each subsequent upregulation phase and the difference expressed as PSC. The PSC for upregulation was averaged per run, leading to nine PSC values per subject. An analysis of variance was then calculated with the PSC as dependent measure and the training run as within-subjects factor. As measure of effect size generalised eta squared $\left(\eta_{G}^{2}\right)$ is reported (43).

As one subject in the healthy control-group aborted the measurement on T3, only one neurofeedback run is present for this subject at that specific time point. Therefore, this subject was excluded from the PSC analysis.

\section{Granger-Causality-Analysis (GCA)}

To assess cerebral connectivity, GCA was performed in R. GCA is an approach to assess effective connectivity (44). Its principle is that given two time series $X$ and $Y, Y$ is said to g-cause $X$ if the future of $\mathrm{X}$ is predicted better incorporating information from $Y$ than from $X$ alone.

Clusters to be used for a pairwise GCA were selected post hoc based on functional results of groups $\mathrm{HC}$ and $\mathrm{PA}$, so that all active regions were part of the analysis. In case a region was active in both groups or multiple clusters of activation were present within one region, only the largest cluster was selected. Clusters below 20 functional voxels in size were not considered for selection. Raw activation time courses were extracted and averaged per training day for each cluster and group. Data were then checked for unit-roots and autocorrelations as well as time courses with high colinearity. The Bayesian Information Criterion was determined to select the lag size for the analysis. Finally, a F-test for Granger-causality was computed for each pairwise combination of entered clusters and p-values were adjusted using the Holm method.

\section{Voxel-Based Morphometry (VBM)}

Voxel-based morphometry of structural MRI data were performed using the CAT12 toolbox (45), implemented in SPM12 (http:// www.fil.ion.ucl.ac.uk/spm/) running on Matlab. As we were interested in GM volume changes from T1 to T5, we used the CAT12 protocol for processing of longitudinal data. Pre-processing steps incorporated intra-subject realignment, bias correction, segmentation, and spatial normalisation. After an initial realignment 
of individual anatomical scans to standardised (MNI) space, the mean of the realigned images was calculated and used as reference image in a subsequent realignment. The realigned individual images were then bias-corrected to account for signal inhomogeneities. The mean image was segmented into GM, white matter and cerebrospinal fluid and normalised using DARTEL. The resulting spatial normalisation parameters were then applied to the segmentations of the bias-corrected individual images of both time points, which were again realigned. To allow comparison of the absolute tissue volume, voxel values were modulated using the Jacobian determinants (i.e., linear and non-linear components) derived from the spatial normalisation. Finally, the modulated GM images were smoothed with a Gaussian kernel of $8 \mathrm{~mm}$ FWHM. Between-group differences in GM volumes at baseline were assessed using two-sample $t$-tests adjusted for total intracranial volume (TIV), age, and gender. In a flexible factorial design with the factors "subject," "group," and "time" and after including TIV as nuisance parameter, main effects of time and group-by-time interactions were tested. To avoid possible edge effects around the border between tissue types, an absolute GM threshold of 0.01 was applied. For voxel-wise statistical analysis, we used a cluster-level family wise error correction of $p<0.05$ across the whole-brain (uncorrected height threshold of $p<0.001$ ) adjusted for non-stationarity (46). Additionally, PHG and hippocampal volumes were derived as ROI, corrected for TIV and compared between groups.

\section{Debriefings}

The data from debriefings after rtfMRI training runs were first converted into numerical scores. For the question whether subjects thought that they were able to voluntarily influence the thermometer bar, the answer no was converted to 0 , partially to 1 and yes to 2 (leading to a maximum total score of 6 per training day). As equidistance between answers could not be assumed, only statistics requiring at most ordinal scale were applied to debriefing data. To assess changes in debriefing response over the course of the training, a Friedman test over all three training days was computed for each experimental group. For analysis of differences between groups Kruskal-Wallis tests were performed.

\section{RESULTS}

\section{Sample}

In total, 10 female and 20 male subjects completed the study (mean age 64.567, SD = 7.838). Of these, seven female and nine male subjects were part of group $\mathrm{HC}$; two female and eight male subjects formed group PA; and one female and three male subjects were part of group SH. There were no differences between groups in age $\left[F(2,27)=0.350, p=0.709, \eta^{2}=0.025\right]$ or BDI-II score $\left[F(2,27)=0.655, p=0.528, \eta^{2}=0.044\right]$. There was a difference in estimated premorbid intelligence between groups $\left[F(2,27)=5.079, p=0.013, \eta^{2}=0.273\right]$, where pairwise $t$-tests suggest that estimated premorbid intelligence was bigger in $\mathrm{HC}$ compared to PA, but no difference between $\mathrm{SH}$ and both other
TABLE 1 | Demographics and characteristics of the sample.

\begin{tabular}{lccc}
\hline & HC & PA & SH \\
\hline$n$ & 16 & 10 & 4 \\
Female & 7 & 2 & 1 \\
Male & 9 & 8 & 3 \\
Mean age & 63.5 & 66.2 & 64.75 \\
$( \pm$ SD; range $)$ & $( \pm 6.663 ; 53-76)$ & $( \pm 8.930 ; 53-80)$ & $( \pm 9.453 ; 51-73)$ \\
\hline
\end{tabular}

\section{Education}

\begin{tabular}{lccc}
\hline Higher education & 7 & 3 & 2 \\
13 years in school & 4 & 2 & 1 \\
12 years in school & 2 & 0 & 0 \\
10 years in school & 3 & 2 & 0 \\
9 years in school & 0 & 2 & 0 \\
8 years in school & 0 & 1 & 1 \\
\hline Psychometrics & & & 6 \\
\hline Mean BDI score & 3.313 & 5.2 & $( \pm 9.416 ; 0-20)$ \\
$\begin{array}{l} \pm \text { SD; range }) \\
\text { Mean MWT-B IQ }\end{array}$ & $( \pm 3.807 ; 0-11)$ & $( \pm 5.203 ; 0-15)$ & 121.5 \\
$( \pm$ SD; range $)$ & $( \pm 9.295 ; 112-136)$ & $( \pm 14.062 ; 91-130)$ & $( \pm 21.734 ; 100-143)$
\end{tabular}

Neurodegeneration markers in cerebrospinal fluid (mean \pm SD; range)

\begin{tabular}{lcc}
\hline $\begin{array}{l}\text { Amyloid } \beta_{1-42} \\
(\mathrm{pg} / \mathrm{ml})\end{array}$ & - & 597.111 \\
Amyloid $\beta_{1-40}$ & - & $( \pm 163.164 ; 409-928)$ \\
$(\mathrm{pg} / \mathrm{ml})$ & & $14,971.125$ \\
$\beta_{1-42} / \beta_{1-40}$ ratio & - & 0.476 \\
& & $( \pm 0.243 ; 0.24-1)$ \\
Total tau $(\mathrm{pg} / \mathrm{ml})$ & - & 367.444 \\
& - & $74.556 .557 ; 95-927)$ \\
Phospho-tau & - & $( \pm 35.606 ; 28-126)$ \\
(pg/ml) & &
\end{tabular}

An overview of certain characteristics of participants is given. Cerebrospinal fluid characteristics were unavailable for one patient, the amyloid $\beta_{1-40}$ value was missing in one additional case. Reference values were used from the Neurochemical Laboratory at the University of Göttingen, Germany: $\beta$-amyloid ${ }_{1-42}$ : $>450 \mathrm{pg} / \mathrm{ml} ; \beta$-amyloid ratio $\left[\left(\beta\right.\right.$-amyloid 1-42 $_{2} / \beta$-amyloid $\left.\left.1-40\right) \times 10\right]>0.5$; total tau protein: $<450 \mathrm{pg} / \mathrm{ml}$; phospho-tau protein $_{181 \mathrm{p}}$ : $>61 \mathrm{pg} / \mathrm{ml}$.

BDI, Beck Depression Inventory II; MWT-B, Multiple-Select-Word-Choice-Test B; IQ, estimated premorbid intelligence quotient.

groups was present. See Table 1 for full demographic details and neurodegeneration markers in group PA.

\section{Neuropsychological Baseline Profile and Changes after rtfMRI Neurofeedback Training}

In groups $\mathrm{HC}$ and $\mathrm{SH}$, average neuropsychological scores were in the normal range in both the pre- (T1) and post-test (T5). In group PA scores of the following tests were below the normal range: MoCA, VVM Verbal Memory immediate recall (T1 only) and delayed recall, WMS Visual Memory delayed recall (T5 only), WMS Verbal Memory delayed recall and TMT-B (see Table 2 for details).

In $\mathrm{HC}$, increases in scores from pre to post-test were found for the visuospatial task of the VVM in the immediate recall condition $[t(15)=-2.690, p=0.017]$, for the MoCA $[t(15)=-3.721$, $p=0.002]$ and the backward digit-span task of the WMS $[t(15)=-2.817, p=0.013]$. Additionally, a trend was found for 
TABLE 2 | Descriptive statistics.

\begin{tabular}{|c|c|c|c|c|c|c|}
\hline \multirow[t]{2}{*}{ Test } & \multicolumn{2}{|c|}{$\mathrm{HC}$} & \multicolumn{2}{|c|}{ PA } & \multicolumn{2}{|c|}{ SH } \\
\hline & Pre & Post & Pre & Post & Pre & Post \\
\hline MoCA & $26.813 \pm 1.974$ & $28 \pm 1.592$ & $24.8 \pm 3.225$ & $24.5 \pm 2.915$ & $26 \pm 4.243$ & $26.25 \pm 3.775$ \\
\hline Visual and Verbal Memory Test (MM) Visp 1 & $53.188 \pm 24.460$ & $69.063 \pm 23.268$ & $38.2 \pm 35.080$ & $60.2 \pm 20.778$ & $79.5 \pm 19.140$ & $79 \pm 16.513$ \\
\hline WM Visp 2 & $50.563 \pm 25.222$ & $59.5 \pm 22.675$ & $24.2 \pm 23.794$ & $48.7 \pm 28.987$ & $59.25 \pm 24.541$ & $66.25 \pm 9.639$ \\
\hline WM Verbal 1 & $49.063 \pm 26.941$ & $51.25 \pm 28.252$ & $17.6 \pm 22.887$ & $19.4 \pm 15.551$ & $67 \pm 13.589$ & $33 \pm 42.166$ \\
\hline WM Verbal 2 & $46.063 \pm 25.878$ & $47.938 \pm 30.163$ & $16.6 \pm 24.496$ & $14.6 \pm 15.472$ & $56.5 \pm 21$ & $36 \pm 31.294$ \\
\hline Wechsler Memory Scale (WMS) Visual 1 & $50.5 \pm 32.922$ & $61.75 \pm 31.298$ & $37.6 \pm 36.855$ & $18.2 \pm 17.781$ & $73.75 \pm 23.614$ & $64.25 \pm 27.945$ \\
\hline WMS Visual 2 & $55.125 \pm 29.209$ & $53.750 \pm 31.792$ & $24.6 \pm 31.224$ & $13.4 \pm 15.072$ & $63 \pm 38.730$ & $49.25 \pm 31.170$ \\
\hline WMS Verbal 1 & $52.933 \pm 29.550$ & $62.2 \pm 26.474$ & $19.9 \pm 23.727$ & $25.9 \pm 25.736$ & $44.75 \pm 38.448$ & $51.75 \pm 34.760$ \\
\hline WMS Verbal 2 & $54.733 \pm 32.017$ & $56.6 \pm 30.016$ & $14.7 \pm 21.145$ & $16 \pm 21.566$ & $42.25 \pm 29.216$ & $31.25 \pm 37.277$ \\
\hline WMS Digit Fw & $77.5 \pm 16.653$ & $71.313 \pm 23.105$ & $49.2 \pm 32.454$ & $42.8 \pm 35.496$ & $59 \pm 25.179$ & $78.5 \pm 17.059$ \\
\hline WMS Digit BW & $59.25 \pm 29.051$ & $72.875 \pm 20.720$ & $45.8 \pm 35.443$ & $58.4 \pm 27.893$ & $49.5 \pm 27.574$ & $61.5 \pm 17.673$ \\
\hline TMT-A & $46.366 \pm 9.132$ & $48.504 \pm 10.885$ & $45.554 \pm 19.283$ & $48.974 \pm 19.215$ & $60.230 \pm 7.366$ & $57.673 \pm 9.794$ \\
\hline TMT-B & $19.596 \pm 14.509$ & $19.464 \pm 13.058$ & $15.424 \pm 20.475$ & $14.952 \pm 16.938$ & $37.5 \pm 25.613$ & $32.098 \pm 23.357$ \\
\hline Visual Patterns Test & $60.625 \pm 30.434$ & $62.813 \pm 29.324$ & $44 \pm 31.605$ & $46 \pm 21.211$ & $53.75 \pm 33.260$ & $73.75 \pm 17.5$ \\
\hline
\end{tabular}

Given is mean $\pm 1 S D$ for each group at each time point. Scores are percent ranks, except the MoCA for which raw scores are shown.

Visp, visuospatial; fw, forward; bw, backward.

Immediate recall is denoted by 1 , delayed recall by 2 .

TABLE 3 | Linear mixed model results for examination time point.

\begin{tabular}{|c|c|c|c|}
\hline Test & $\mathrm{HC}$ & PA & SH \\
\hline $\begin{array}{l}\text { Visual and Verbal Memory Test } \\
\text { (WM) Visp } 1\end{array}$ & $t=-2.690, p=0.017, \Omega_{0}^{2}=0.679$ & $t=-2.187, p=0.057, \Omega_{0}^{2}=0.642$ & $t=0.044, p=0.968, \Omega_{0}^{2}=0.337$ \\
\hline WM Visp 2 & $t=-1.864, p=0.082, \Omega_{0}^{2}=0.809$ & $t=-3.041, p=0.014, \Omega_{0}^{2}=0.768$ & $t=-0.780, p=0.492, \Omega_{0}^{2}=0.732$ \\
\hline WM Verbal 1 & $t=-0.353, p=0.729, \Omega_{0}^{2}=0.733$ & $t=-0.214, p=0.835, \Omega_{0}^{2}=0.228$ & $t=1.441, p=0.245, \Omega_{0}^{2}=0.321$ \\
\hline WM Verbal 2 & $t=-0.419, p=0.681, \Omega_{0}^{2}=0.887$ & $t=0.262, p=0.799, \Omega_{0}^{2}=0.504$ & $t=1.945, p=0.318, \Omega_{0}^{2}=0.515$ \\
\hline MoCA & $t=-3.721, p=0.002, \Omega_{0}^{2}=0.873$ & $t=1.152, p=0.279, \Omega_{0}^{2}=0.982$ & $t=-0.333, p=0.761, \Omega_{0}^{2}=0.964$ \\
\hline $\begin{array}{l}\text { Wechsler Memory Scale (WMS) } \\
\text { Verbal } 1\end{array}$ & $t=-1.197, p=0.252, \Omega_{0}^{2}=0.866$ & $t=-0.708, p=0.497, \Omega_{0}^{2}=0.616$ & $t=-0.392, p=0.721, \Omega_{0}^{2}=0.609$ \\
\hline WMS Verbal 2 & $t=-0.066, p=0.949, \Omega_{0}^{2}=0.868$ & $t=-0.194, p=0.850, \Omega_{0}^{2}=0.685$ & $t=0.522, p=0.638, \Omega_{0}^{2}=0.362$ \\
\hline WMS Visual 1 & $t=-1.430, p=0.173, \Omega_{0}^{2}=0.699$ & $t=2.187, p=0.056, \Omega_{0}^{2}=0.735$ & $t=0.713, p=0.528, \Omega_{0}^{2}=0.577$ \\
\hline WMS Visual 2 & $t=0.257, p=0.800, \Omega_{0}^{2}=0.862$ & $t=1.450, p=0.181, \Omega_{0}^{2}=0.701$ & $t=1.794, p=0.171, \Omega_{0}^{2}=0.950$ \\
\hline WMS Digit FW & $t=1.655, p=0.119, \Omega_{0}^{2}=0.831$ & $t=0.523, p=0.614, \Omega_{0}^{2}=0.557$ & $t=-2.832, p=0.066, \Omega_{0}^{2}=0.891$ \\
\hline WMS Digit Bw & $t=-2.817, p=0.013, \Omega_{0}^{2}=0.841$ & $t=-1.667, p=0.130, \Omega_{0}^{2}=0.848$ & $t=-1.260, p=0.297, \Omega_{0}^{2}=0.831$ \\
\hline Visual Patterns Test & $t=-0.353, p=0.730, \Omega_{0}^{2}=0.787$ & $t=-0.263, p=0.799, \Omega_{0}^{2}=0.770$ & $t=-1.414, p=0.252, \Omega_{0}^{2}=0.701$ \\
\hline TMT-A & $t=-0.781, p=0.447, \Omega_{0}^{2}=0.583$ & $t=-1.022, p=0.333, \Omega_{0}^{2}=0.919$ & $t=0.502, p=0.650, \Omega_{0}^{2}=0.488$ \\
\hline TMT-B & $t=0.034, p=0.974, \Omega_{0}^{2}=0.518$ & $t=0.257, p=0.802, \Omega_{0}^{2}=0.976$ & $t=0.514, p=0.626, \Omega_{0}^{2}=0.805$ \\
\hline
\end{tabular}

Shown are results for differences in examination time points for all neuropsychological tests in all groups. Additionally the overall model fit is given. Models may include the additional predictors gender (all except for TMT-A/B) and age (all WMS subtests), where the normative data did not respect these variables (data not shown).

Visp, visuospatial memory; fw, forward; bw, backward.

1 , immediate recall; 2 , delayed recall.

an increase of scores in the delayed recall condition of the visuospatial memory task of the VVM $[t(15)=-1.864, p=0.082]$.

Group PA displayed an increase in scores for the delayed recall of the visuospatial memory task of the VVM $[t(9)=-3.041$, $p=0.014]$. A trend for an increase of scores in the immediate recall of that task $[t(9)=-2.187, p=0.057]$ and a further trend for a decrease in scores for immediate recall condition of the WMS visual memory task $[t(9)=2.188, p=0.056]$ were present.
No changes in scores from pre to post-test were found in $\mathrm{SH}$. An overview of all models is given in Table 3.

\section{Brain Activation during Training}

In groups $\mathrm{HC}$ and $\mathrm{PA}$, activation of the target region (left PHG) was revealed, but not in SH (see Table 4 and Figure 3). In HC, activation was further found in the right precuneus, left posterior cingulate, right PHG, bilaterally in the superior occipital gyrus and middle frontal gyrus, as well as in cerebellar areas. Averaged 
TABLE 4 | Brain activation averaged across all neurofeedback runs.

\begin{tabular}{|c|c|c|c|c|c|c|c|}
\hline Region & BA & $k$ & $x$ & $y$ & $z$ & $T_{\max }$ & $T_{\text {mean }}$ \\
\hline \multicolumn{8}{|c|}{ Group HC, $p<10^{-6}, k>2, \mathrm{df}=3,727$} \\
\hline R Precuneus & 31,7 & 855 & 16 & -62 & 18 & 15.126 & 7.323 \\
\hline \multicolumn{8}{|l|}{ gyrus (PHG) } \\
\hline $\mathrm{R} P H G$ & & 101 & 27 & -31 & 4 & 11.882 & 7.397 \\
\hline L Superior occipital gyrus & 19 & 164 & -37 & -75 & 23 & 10.767 & 6.948 \\
\hline R Superior occipital gyrus & 39 & 105 & 39 & -73 & 20 & 10.429 & 6.518 \\
\hline L Middle frontal gyrus & 6 & 110 & -29 & -2 & 46 & 8.077 & 5.748 \\
\hline R Cerebellar anterior lobe & & 20 & 11 & -40 & -27 & 6.984 & 5.813 \\
\hline R Middle frontal gyrus & 6 & 32 & 27 & -6 & 48 & 6.764 & 5.571 \\
\hline R Declive & & 11 & 15 & -62 & -18 & 6.024 & 5.294 \\
\hline R Declive & & 16 & 15 & -80 & -11 & 6.019 & 5.357 \\
\hline R Cerebellar tonsil & & 20 & 37 & -54 & -32 & 5.739 & 5.269 \\
\hline L Middle Frontal gyrus & 9 & 12 & -40 & 17 & 31 & 5.695 & 5.252 \\
\hline L Cerebellar anterior lobe & & 9 & -7 & -40 & -25 & 5.647 & 5.229 \\
\hline R Culmen & & 10 & 30 & -51 & -21 & 5.480 & 5.177 \\
\hline L Medial frontal gyrus & 6 & 2 & -11 & 2 & 56 & 5.251 & 5.082 \\
\hline L Cingulate gyrus & 31 & 4 & -16 & -40 & 34 & 5.208 & 5.185 \\
\hline L Middle frontal gyrus & 47 & 4 & -35 & 38 & -2 & -5.452 & -5.377 \\
\hline R Precentral gyrus & 4 & 3 & 54 & -13 & 40 & -5.835 & -5.221 \\
\hline \multicolumn{8}{|c|}{ Group PA, $p<0.001, k>16, \mathrm{df}=2,329$} \\
\hline L Posterior cingulate & 29 & 25 & 12 & -50 & 13 & 4.644 & 3.806 \\
\hline L Precuneus & 7 & 31 & -3 & -60 & 36 & 3.784 & 3.582 \\
\hline \multicolumn{8}{|c|}{ Group SH, $p<0.001, k>23, \mathrm{df}=931$} \\
\hline & & & & & & & \\
\hline
\end{tabular}

A contrast for upregulation > baseline was computed for each run and then data were averaged across all runs for each group. Size thresholds were determined using Alphasim. BA, Brodmann area; $k$, cluster size ( $3 \mathrm{~mm}^{3}$ voxels); $x / y / z$, peak coordinate in Talairach space; $T_{\max }$ and $T_{\text {mean, }}$ peak and average $T$-value of the cluster; $d$, degrees of freedom.

over the course of the training, in group PA activation was only found in the left precuneus and right posterior cingulate. A small cluster within the left PHG is below cluster-size threshold. On T3 only, broader activation was found including the left precuneus, bilateral middle frontal gyrus, left medial frontal gyrus, right cerebellar tonsil, left precentral gyrus, left middle occipital gyrus and left parahippocampus (see Table 5).

Analysis of changes in left PHG activation during upregulation phases expressed as PSC yielded no significant results in group $\mathrm{HC}\left[F(8,112)=0.992, p=0.446, \eta_{G}^{2}=0.017\right]$. The same is true for groups PA $\left[F(8,72)=1.624, p=0.133, \eta_{G}^{2}=0.065\right]$ and SH $\left[F(8,24)=0.392, p=0.914, \eta_{G}^{2}=0.112\right]$. When comparing differences between groups, a trend was found $[F(2,26)=2.954$, $\left.p=0.070, \eta_{G}^{2}=0.132\right]$ indicating $\mathrm{HC}>\mathrm{PA}>\mathrm{SH}$. No differences over time $\left[F(8,208)=1.246, p=0.274, \eta_{G}^{2}=0.016\right]$ and no interaction between group and time $[F(16,208)=1.106, p=0.351$, $\left.\eta_{G}^{2}=0.028\right]$ were revealed. See Figure 4 for a visualisation of PSC in upregulation phases over time in all groups.

\section{Granger-Causality-Analysis}

Based on functional results, 11 clusters were selected for GCA: right precuneus, left posterior cingulate, left and right PHG, left and right middle frontal gyrus, left and right superior occipital gyrus, left superior temporal gyrus (temporo-parietal-junction), right cerebellar tonsil and right anterior cerebellar lobe.
The networks are visualised in Figure 5. The most important findings are that in $\mathrm{HC}$ the left PHG appears to change its role to a mainly receiving, but less driving region with an increasing number of inputs and a decreasing number of outputs over the course of the training. In contrast the opposite pattern can be observed for the right precuneus in $\mathrm{HC}$, which becomes mainly a driver of activation.

Similar results were found for group PA regarding the right precuneus, but different than in $\mathrm{HC}$ the role of the left PHG remained unchanged across all three training days.

\section{Voxel-Based Morphometry}

Region of interest-based analysis revealed significant GM volume loss at T1 in PA compared to HC in the left PHG [one-sided $t$-test: $t(16.095)=2.110, p=0.025]$ and a trend for the left hippocampus $[t(10.663)=1.508, p=0.080]$. Nonetheless, voxel-wise comparison of initial GM volumes between groups HC and PA did not yield any significant differences across the whole brain.

When comparing changes over time increases in GM volumes across both groups were found in two locations: the right precuneus (541 vx; $T=4.77, p=0.038$; MNI 6/-56/21, TAL approx. $6 /-53 / 22)$ and the right superior medial frontal gyrus (261vx; $T=5.87, p=0.048 ;$ MNI 9/30/40, TAL approx. 9/31/35). Further trends were found for the middle occipital gyrus $(349 \mathrm{vx} ; T=4.85$, $p=0.052$; MNI 36/-76/21, TAL approx. 36/-73/23) and the middle frontal gyrus (163vx; $T=5.91, p=0.074 ; \mathrm{MNI} 38 / 16 / 54$, TAL approx. 38/18/49). Neither decreases, nor interactions between experimental groups and time were found.

\section{Debriefing after rtfMRI Neurofeedback Training}

Group HC had an overall median debriefing score of $2(\mathrm{MAD}=0)$. This was lower for group PA with a median of $1(\mathrm{MAD}=0.5)$ and group $\mathrm{SH}$ with a median score of $1(\mathrm{MAD}=0.5)$. The analysis of the subjective rating over the course of time did not suggest any changes for group $\mathrm{HC}\left[\chi^{2}(8)=4.188, p=0.840\right]$; neither for group $\mathrm{PA}\left[\chi^{2}(8)=4.636, p=0.769\right]$; nor for group $\mathrm{SH}\left[\chi^{2}(8)=2.222\right.$, $p=0.973]$.

A comparison between all three groups revealed significant differences, though $\left[\chi^{2}(2)=12.684, p=0.002\right]$. Pairwise KruskalWallis tests showed that this effect was caused by larger scores in group $\mathrm{HC}$ compared to each of the other two groups [HC vs. PA: $\chi^{2}(1)=9.561, p=0.002$; HC vs. SH: $\left.\chi^{2}(1)=6.436, p=0.011\right]$. Scores between PA and SH were not different $\left[\chi^{2}(1)=0.545\right.$, $p=0.461]$.

\section{DISCUSSION}

The present study applied rtfMRI neurofeedback training targeting the left PHG and visuospatial memory in healthy elderly and patients of $\mathrm{pAD}$ with the aim to improve cognitive performance. Results indicate that the training improved the targeting domain of visuospatial memory in elderly as well as patients. However, in $\mathrm{pAD}$ improvement in the target domain was accompanied by decreased performance in a visual memory task. A small group receiving feedback of a brain region unrelated to the paradigm, but 

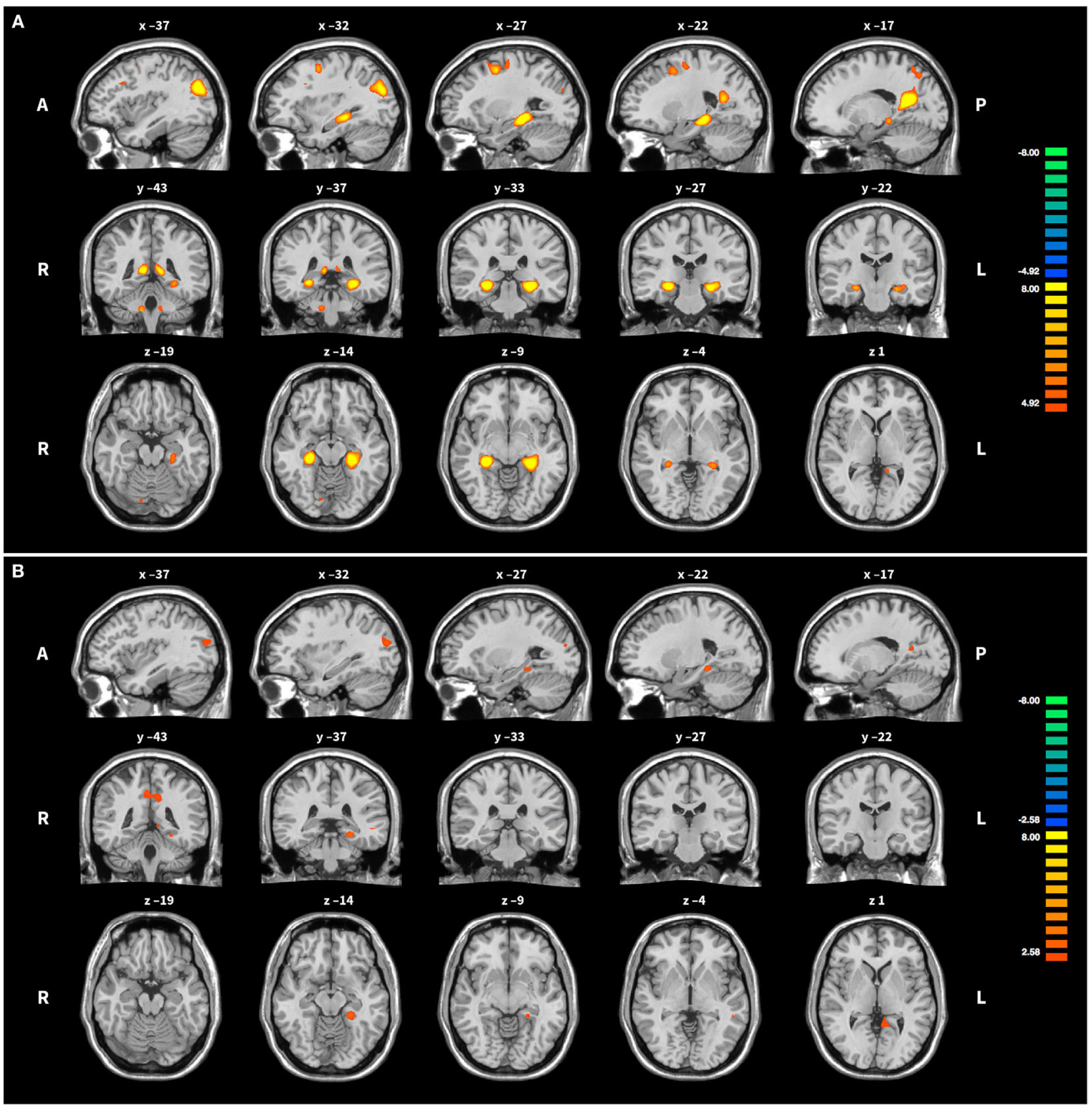

FIGURE 3 | Activation during upregulation. (A) Group HC: the middle column shows the peak of activation in the left parahippocampal gyrus, the target region of the training. Some further activation in frontal, parietal and cerebellar areas is visible as well. (B) Group PA: for easier comparison slices shown are the same as for group HC. Note the more liberal activation threshold in group PA. For both groups activation was averaged across all training runs and superimposed onto the Colin 27 average brain. Copyright (c) 1993-2009 Louis Collins, McConnell Brain Imaging Centre, Montreal Neurological Institute, McGill University.

otherwise performing the same task, did not display any changes in cognitive performance from pre to post-test. In the healthy control group and in pAD patients GCA suggested changes in networks affecting the parahippocampus and precuneus. Voxelbased morphometry showed structural changes in the precuneus, frontal and occipital areas after training. Generally, this proofof-concept study is in line with previous rtfMRI neurofeedback training studies (17), but extending the literature with evidence for feasibility and possibly successful application of rtfMRI neurofeedback training to patients with $\mathrm{pAD}$.

The strongest indicator that the conducted training led to the intended outcome is the result of the VVM visuospatial memory test. This measure represents cognitive abilities of the targeted domain; therein we found improvements in groups HC and PA but not in SH. Presence of effects only for groups that underwent training of the PHG suggests that mental imagery alone 
TABLE 5 | Brain activation during neurofeedback on T3 in group PA.

\begin{tabular}{lrrrrrrr}
\hline Region & BA & $\boldsymbol{k}$ & $\boldsymbol{x}$ & $\boldsymbol{y}$ & $\boldsymbol{z}$ & $\boldsymbol{T}_{\text {max }}$ & $\boldsymbol{T}_{\text {mean }}$ \\
\hline L Precuneus & 7 & 487 & 0 & -57 & 38 & 5.491 & 3.990 \\
L Medial Frontal Gyrus & 8 & 53 & -12 & 28 & 38 & 5.018 & 3.788 \\
L Precentral Gyrus & 6 & 37 & -47 & 4 & 34 & 4.776 & 3.770 \\
L Parahippocampal Gyrus & & 24 & -27 & -36 & 5 & 4.607 & 3.849 \\
R Middle Frontal Gyrus & 9 & 40 & 43 & 15 & 29 & 4.409 & 3.667 \\
L Superior Temporal Gyrus & 39 & 23 & -48 & -56 & 21 & 4.398 & 3.579 \\
L Middle Occipital Gyrus & 19 & 28 & -31 & -83 & 16 & 4.230 & 3.686 \\
R Cerebellar Tonsil & & 36 & 25 & -65 & -32 & 4.176 & 3.555 \\
L Middle Frontal Gyrus & 6 & 32 & -36 & 14 & 43 & 4.059 & 3.662 \\
R Middle Occipital Gyrus & 19 & 22 & 40 & -72 & 11 & 4.032 & 3.559 \\
L Middle Temporal Gyrus & 21 & 22 & -53 & -44 & 6 & 3.921 & 3.553 \\
L Middle Frontal Gyrus & 6 & 17 & -27 & 1 & 48 & 3.802 & 3.486
\end{tabular}

As a peak in activation was present on T3 more activation is found here during upregulation phases than on the other two training days. Data are thresholded to $p<0.001$ and $k>16$; $d f=2,329 . B A$, Brodmann area; $k$, cluster size ( $3 \mathrm{~mm}^{3}$ voxels); $x / y / z$, peak coordinate in Talairach space; $T_{\max }$ and $T_{\text {mean, }}$ peak, and average $T$-value of the cluster; $d f$, degrees of freedom.

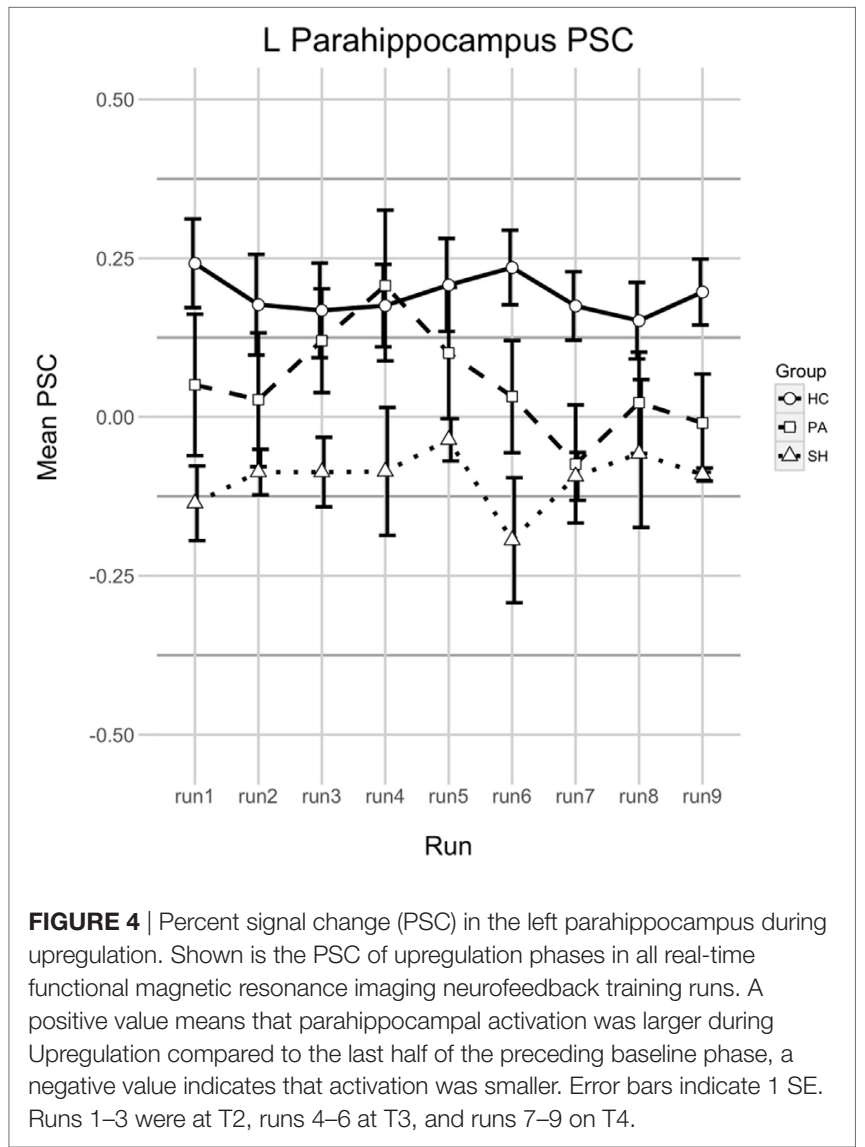

is insufficient to elicit these effects, and only reliable feedback of the PHG leads to improvements. The concept of mental imagery training alone inducing effects is not novel and paradigms with positive outcomes after mental imagery training have for example been reported in various settings [see, for example, Ref. (47-49)].

The course of parahippocampal activation throughout the training did not reflect behavioural changes in test performance over time. For group HC, PSC of PHG activation during upregulation phases remained stable over the course of the training. For SH PSC of PHG was stable as well, but negative indicating less activation during upregulation than baseline. This fits the unrelated target region and lack of behavioural changes well. While failing statistical significance, patterns of PSC were different for group PA which (1) showed overall lower PSC than group $\mathrm{HC}$, which is in line with findings of decreased memoryrelated activation in $\mathrm{AD}$ in that region (50); (2) despite overall low PSC showed a strong-but not statistically significant-peak on the first feedback run on T3 and (3) displayed mainly negative PSC on T4 suggesting difficulties to perform the task on that day. It remains unclear, why the course of PSC was fluctuating this much in group PA. However, the lower PSC levels compared to $\mathrm{HC}$ and the apparent problems in properly performing the task on the last training day seem to fit the fewer increases in behavioural performance in this group compared to HC.

The literature is inconclusive regarding whether an increase or a decrease in brain activation is related to increased cognitive performance. On the one hand, increased activation is often interpreted as translating into increased performance $(12,51)$; on the other hand decreased activation is usually being explained as increased efficiency also translating into improved function $(52,53)$. Given the lack of significant changes in PSC in groups $\mathrm{HC}$ and PA it seems more plausible to assume that the training lead to increased efficiency. A critical point when looking at previous rtfMRI neurofeedback training studies is that many studies reported a clear trend of activation in the target region over the course of the training [see for example $(20,23,54)$ ]. Future studies should shed light on the circumstances under which an increase in PSC during the target condition is established and under which circumstances it remains constant.

For all neuropsychological tests beyond the visuospatial memory task of the VVM for which improvements were found in $\mathrm{HC}$, evidence has been reported that the cognitive domains these tests measure are related to the PHG. For working memory, as measured by the backward digit-span task, this is true in cases in which novel information is kept in working memory (55-57), but not in other cases $(58,59)$. It is certainly debatable whether the task is novel enough to elicit PHG involvement, but given the present results it appears plausible. The issue of PHG involvement is clearer for the MoCA, as many of its subtasks have been reported as being related to the PHG. As detailed above, the task on delayed recall and visuospatial processing target domains associated with the PHG. Furthermore, associations between the PHG and verbal abstraction tasks have also been reported in the literature (60).

The issue of worse performance in the WMS visual memory task in group PA is more difficult to explain. The adoption of techniques and strategies that enhance performance in one cognitive domain but impair another, suggesting negative transfer $(61,62)$, has been recently reported (63). There is not much further literature on this matter, especially not in the context of rtfMRI neurofeedback training.

Functional MRI and VBM results generally fit the overall concept of the training task and its demands. Activation that was found during upregulation phases represents visuo-motor imagery and retrieval of episodic memory contents well. The substantially less activation that was found in group PA 


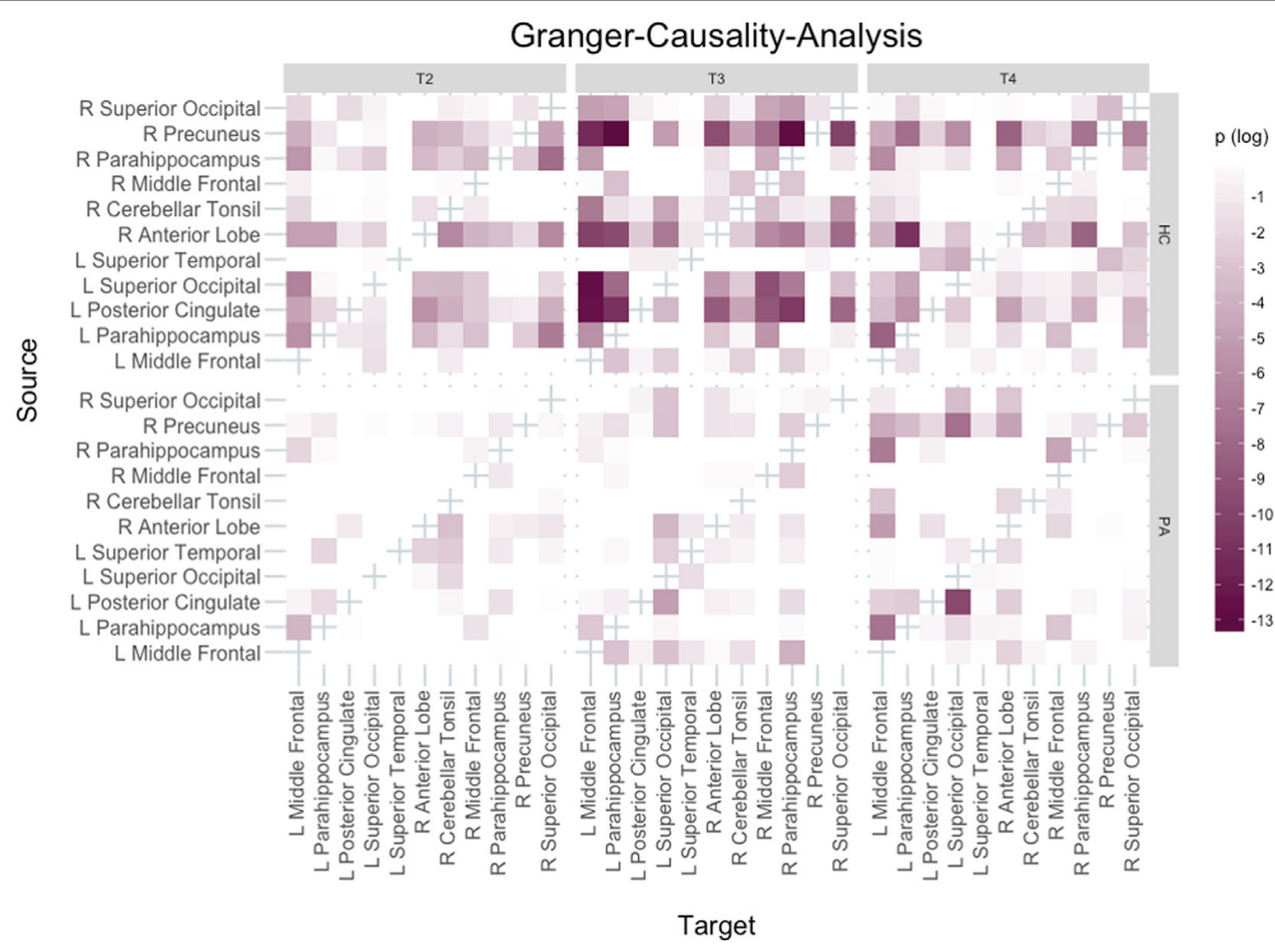

FIGURE 5 | Granger-Causality-Analysis results. Directionality of Granger-causality is from source to target region. Bonferroni-Holm adjusted $p$-values were logarithmised with base 10, i.e., the darker a tile the smaller the $p$-value.

compared to HC, can be well explained by the expected difficulties in recall of memories in group PA. There is conflicting evidence regarding the overall change of activation in $\mathrm{AD}$ (64), so it is difficult to conclude whether the activation found in group PA is due to difficulties in performing the task or due to the effects of pAD. As expected, no PHG activation and overall low activation was found in SH. A critical point here may be the small sample size making broad activation less likely to be found, but the results of the PSC analysis show that activation was indeed lower during upregulation phases than during baseline in group SH. A possibility here may be that generation of numerous different strategies for modulation of left PHG activation due to the unresponsive feedback stimulus hampers broad activation and consequently enhanced cognitive performance.

Differences in GM volume between groups were found in the left hippocampus and parahippocampus. These are regions associated with $\mathrm{AD}$ and a loss of GM in PA in these regions thus expected. The lack of a group difference in the whole-brain analysis seems unusual, though, but is not implausible given both the prodromal state of the disease and the small group size. Longitudinally on the whole-brain level, while no changes in left PHG volume were found, the finding of increased GM volume in the right precuneus fits well into the data. Large clusters of activation were found for that region during upregulation phases of rtfMRI neurofeedback training and that region has been associated with memory retrieval, visuo-motor imagery and aspects of consciousness $(65,66)$. It has also been hypothesised that the parietal lobe and especially the precuneus might be critical for $\mathrm{AD}(67,68)$ suggesting that for the conducted training the precuneus is an essential region and a GM volume increase therein is especially promising for $\mathrm{AD}$. Moreover, increased $\mathrm{GM}$ volume in the middle occipital gyrus fits well into the present paradigm, as a core component of it was mental imagery of a previously memorised real-world footpath. Finally, also the findings for both frontal regions can be explained well in the context of the training. The medial location has been associated with cognitive control $(69,70)$ which is a cognitive component required to monitor the state of the feedback stimulus and to adapt strategies accordingly. The middle frontal location is in contrast associated with working memory (71), a cognitive component which is very relevant for the training task as the state of the feedback stimulus not only needs to be monitored, but also compared to previous states, to successfully apply modulation strategies. Effects on GM volume induced by cognitive training over the course of a few weeks have been demonstrated recently (72).

Granger-causality-analysis results suggest that both in group $\mathrm{HC}$ and group PA the precuneus becomes the main driver of activation. There are some reports of the precuneus playing a role for feedback learning $(73,74)$, but none in the context of a paradigm comparable to the present study. The possible involvement of the precuneus in feedback learning, together with its associations elaborated on above, seems plausible to explain its changed role 
here. Together with the VBM results this points out the importance of this region for this kind of training. The role of the target region is also very interesting and differing between groups. In group $\mathrm{HC}$, the left PHG seems to become less of a driver of activation over the course of the training, while in group PA it does neither receive many inputs nor is it the source of many connections.

It should be noted that the application of GCA to fMRI data has been subject of debate $(75,76)$. Main points raised against the application of GCA to fMRI data are (1) the low temporal resolution of such data leading to interactions between regions impossible to be displayed; and (2) the fact that the haemodynamic response latency differs between regions of the brain and thus might confound data. There is evidence though, that given met prerequisites and properly formulated research questions GCA on fMRI can provide useful results $(77,78)$. Nevertheless it should be kept in mind that connections faster than the selected lag size are missed entirely. General issues of correlative analyses such as the problem of potential third variables affecting the data apply as well.

As control condition we used a sham-feedback approach with the presentation of feedback from a brain region unrelated to the task (79). A problem with this approach is that subjects may find the strategies they apply have no influence whatsoever on the feedback and thus become frustrated and demotivated. On the one hand, the finding of no cognitive improvement in group $\mathrm{SH}$ in the present work is evidence for the effectiveness of the training paradigm, on the other hand caution is advised in the interpretation of the results as the performance in group SH may have been negatively influenced by motivation, which is known to moderate cognitive training outcome (80). Future research should aim to develop control conditions that explicitly target the motivational problem.

Difficulties in influencing the feedback stimulus are also a concern for the patient group. Subjective ratings of the ability to influence the feedback thermometer indicate that subjects of group PA faced similar difficulties as group $\mathrm{SH}$ despite receiving true feedback of the PHG, implying the same considerations regarding motivation. As it has been suggested previously that a certain intactness of cognitive functioning is required for successful applications of neurofeedback and brain-computer interface paradigms (81), such implementations in the future should be applied as early as possible in the course of the disease to ensure optimal results.

\section{Limitations}

This study has some limitations. First, although the spatial navigation task was standardised as much as possible, it took place in a dynamic, constantly changing real-world environment leading to slight differences in paths between subjects. Another limitation is the small sample size and reduced power of analyses in group $\mathrm{SH}$, so subtle effects could not be detected and the interpretability of neuropsychological results from this group is rather limited. Finally, no follow-up assessment has been done to test whether observed effects persist. There are reports of EEG-neurofeedback effects being present after months to years after the initial training (82-85). Not much work has been done in this regard for fMRI-based neurofeedback [examples of studies with follow-up term-assessments are Ref. $(19,86,87)]$.

\section{CONCLUSION}

In summary, this proof-of-concept study suggests that rtfMRI neurofeedback training paradigms are feasible in patients of pAD and in the healthy elderly and may be used to counteract cognitive effects of both $\mathrm{AD}$ and healthy ageing. As a small group of healthy subjects receiving sham-feedback only did not display PHG activation or cognitive changes over time, it is concluded that mental imagery alone is insufficient to achieve the effects observed in the groups receiving feedback relevant to the task. Neurofeedback training yielded an activation of the target region PHG in elderly and pAD, which was associated with changes of connectivity and GM volume. Future research needs to address the issues of task difficulty in AD patients and of potential transfer effects on untrained cognitive domains. Overall, our results are promising regarding future clinical applications of rtfMRI neurofeedback training as a tool to slow down cognitive decline.

\section{ETHICS STATEMENT}

After full introduction of the study, all subjects gave written informed consent in accordance with the Declaration of Helsinki. The protocol was approved by the local institutional review board at RWTH Aachen University (local reference number EK 049/11).

\section{AUTHOR CONTRIBUTIONS}

$\mathrm{CH}$ took part in recruiting subjects and acquiring data, carrying out data analysis except Voxel-Based-Morphometry, interpreting the data, and writing of the manuscript. NN, HK, and SK took part in recruiting subjects and acquiring data as well as designing the study. ID carried out Voxel-Based-Morphometry, wrote the sections on Voxel-Based-Morphometry of the manuscript, and revised the first draft of the manuscript. CM took part in recruiting subjects and acquiring data. FP contributed to data acquisition and Voxel-Based-Morphometry. RG and AH contributed to setting up the real-time fMRI paradigm and provided assistance with fMRI analysis. NS contributed to the design of the study and provided MR infrastructure. JS contributed to the design of the study. MR took part in study design, data interpretation, and revised the first draft of the manuscript. KR contributed to the design of the study, acquired funding, facilitated recruitment of patients, took part in interpretation of data, and revised the drafts of the manuscript. All authors read and approved the final manuscript.

\section{ACKNOWLEDGMENTS}

The authors gratefully acknowledge all participants for their enduring collaboration and interest in this research. The authors thank the Brain Imaging Facility of the IZKF Aachen for providing technical support. Also, the authors would like to thank the staff from the INM-4 of the Research Centre Jülich and everyone else who helped out with various issues and technical problems regarding the study. Also the authors thank Dr. Zaheer Abbas, Julia Heller, Shahram Mirzazade, and Dr. Sandro Romanzetti for supporting the measurements. 


\section{FUNDING}

The project and the position of $\mathrm{CH}$ were funded by the Alzheimer Forschung Initiative e.V. (AFI 13812 to KR). KR was further

\section{REFERENCES}

1. Ferri CP, Prince M, Brayne C, Brodaty H, Fratiglioni L, Ganguli M, et al. Global prevalence of dementia: a Delphi consensus study. Lancet (2005) 366:2112-7. doi:10.1016/S0140-6736(05)67889-0

2. Ubhi K, Masliah E. Alzheimer's disease: recent advances and future perspectives. J Alzheimers Dis (2013) 33(Suppl 1):S185-94. doi:10.3233/ jad-2012-129028

3. Ballard CG, Mohan RNC, Bannister C, Handy S, Patel A. Wandering in dementia sufferers. Int J Geriatr Psychiatry (1991) 6:611-4. doi:10.1002/ gps.930060811

4. Cherrier MM, Mendez M, Perryman K. Route learning performance in Alzheimer disease patients. Neuropsychiatry Neuropsychol Behav Neurol (2001) 14:159-68.

5. Benke T, Karner E, Petermichl S, Prantner V, Kemmler G. Neuropsychological deficits associated with route learning in Alzheimer disease, MCI, and normal aging. Alzheimer Dis Assoc Disord (2014) 28:162-7. doi:10.1097/ WAD.0000000000000009

6. Aminoff EM, Kveraga K, Bar M. The role of the parahippocampal cortex in cognition. Trends Cogn Sci (2013) 17:379-90. doi:10.1016/j.tics.2013.06.009

7. Trivedi MA, Wichmann AK, Torgerson BM, Ward MA, Schmitz TW, Ries ML, et al. Structural MRI discriminates individuals with mild cognitive impairment from age-matched controls: a combined neuropsychological and voxel based morphometry study. Alzheimers Dement (2006) 2:296-302. doi:10.1016/j. jalz.2006.06.001

8. Lim T-S, Iaria G, Moon SY. Topographical disorientation in mild cognitive impairment: a voxel-based morphometry study. J Clin Neurol (2010) 6:204-11. doi:10.3988/jcn.2010.6.4.204

9. Braak H, Braak E. Neuropathological stageing of Alzheimer-related changes. Acta Neuropathol (1991) 82:239-59. doi:10.1007/BF00308809

10. Hedden T, Gabrieli JDE. Insights into the ageing mind: a view from cognitive neuroscience. Nat Rev Neurosci (2004) 5:87-96. doi:10.1038/nrn1323

11. Gazova I, Laczó J, Rubinova E, Mokrisova I, Hyncicova E, Andel R, et al. Spatial navigation in young versus older adults. Front Aging Neurosci (2013) 5:1-8. doi:10.3389/fnagi.2013.00094

12. Kirchhoff BA, Anderson BA, Barch DM, Jacoby LL. Cognitive and neural effects of semantic encoding strategy training in older adults. Cereb Cortex (2012) 22:788-99. doi:10.1093/cercor/bhr129

13. Hotting K, Holzschneider K, Stenzel A, Wolbers T, Roder B. Effects of a cognitive training on spatial learning and associated functional brain activations. BMC Neurosci (2013) 14:73. doi:10.1186/1471-2202-14-73

14. Moro V, Condoleo MT, Valbusa V, Broggio E, Moretto G, Gambina G. Cognitive stimulation of executive functions in mild cognitive impairment: specific efficacy and impact in memory. Am J Alzheimers Dis Other Demen (2014) 30:153-64. doi:10.1177/1533317514539542

15. Lampit A, Hallock H, Suo C, Naismith SL, Valenzuela M. Cognitive traininginduced short-term functional and long-term structural plastic change is related to gains in global cognition in healthy older adults: a pilot study. Front Aging Neurosci (2015) 7:14. doi:10.3389/fnagi.2015.00014

16. Engvig A, Fjell AM, Westlye LT, Skaane NV, Dale AM, Holland D, et al. Effects of cognitive training on gray matter volumes in memory clinic patients with subjective memory impairment. J Alzheimers Dis (2014) 41:779-91. doi:10.3233/JAD-131889

17. Ruiz S, Buyukturkoglu K, Rana M, Birbaumer N, Sitaram R. Real-time fMRI brain computer interfaces: self-regulation of single brain regions to networks. Biol Psychol (2014) 95:4-20. doi:10.1016/j.biopsycho.2013.04.010

18. Weiskopf N. Real-time fMRI and its application to neurofeedback. Neuroimage (2012) 62:682-92. doi:10.1016/j.neuroimage.2011.10.009

19. Subramanian L, Hindle JV, Johnston S, Roberts MV, Husain M, Goebel R, et al. Real-time functional magnetic resonance imaging neurofeedback for treatment of Parkinson's disease. J Neurosci (2011) 31:16309-17. doi:10.1523/ jneurosci.3498-11.2011 partly funded by the German Federal Ministry of Education and Research (BMBF 01GQ1402). The funding agencies had no influence on study design; collection, analysis, and interpretation of the data; or writing of the manuscript.

20. Subramanian L, Morris MB, Brosnan M, Turner DL, Morris HR, Linden DEJ Functional magnetic resonance imaging neurofeedback-guided motor imagery training and motor training for Parkinson's disease: randomized trial. Front Behav Neurosci (2016) 111:1-13. doi:10.3389/fnbeh.2016. 00111

21. Haller S, Birbaumer N, Veit R. Real-time fMRI feedback training may improve chronic tinnitus. Eur Radiol (2010) 20:696-703. doi:10.1007/ s00330-009-1595-Z

22. Young KD, Zotev V, Phillips R, Misaki M, Yuan H, Drevets WC, et al. Realtime FMRI neurofeedback training of amygdala activity in patients with major depressive disorder. PLoS One (2014) 9:e88785. doi:10.1371/journal. pone.0088785

23. Zhang G, Yao L, Zhang H, Long Z, Zhao X. Improved working memory performance through self-regulation of dorsal lateral prefrontal cortex activation using real-time fMRI. PLoS One (2013) 8:e73735. doi:10.1371/journal. pone. 0073735

24. Sherwood MS, Kane JH, Weisend MP, Parker JG. Enhanced control of dorsolateral prefrontal cortex neurophysiology with real-time functional magnetic resonance imaging (rt-fMRI) neurofeedback training and working memory practice. Neuroimage (2016) 124(Pt A):214-23. doi:10.1016/j. neuroimage.2015.08.074

25. Ghaziri J, Tucholka A, Larue V, Blanchette-Sylvestre M, Reyburn G, Gilbert G, et al. Neurofeedback training induces changes in white and gray matter. Clin EEG Neurosci (2013) 44:265-72. doi:10.1177/1550059413476031

26. Dubois B, Feldman HH, Jacova C, Cummings JL, Dekosky ST, BarbergerGateau P, et al. Revising the definition of Alzheimer's disease: a new lexicon. Lancet Neurol (2010) 9:1118-27. doi:10.1016/S1474-4422(10)70223-4

27. Oldfield RC. The assessment and analysis of handedness: the Edinburgh inventory. Neuropsychologia (1971) 9:97-113. doi:10.1016/0028-3932(71)90067-4

28. Morris JC, Heyman A, Mohs RC, Hughes JP, van Belle G, Fillenbaum G, et al. The consortium to establish a registry for Alzheimer's disease (CERAD). Part I. Clinical and neuropsychological assessment of Alzheimer's disease. Neurology (1989) 39:1159-65. doi:10.1212/WNL.39.9.1159

29. World Medical Association. World Medical Association Declaration of Helsinki: ethical principles for medical research involving human subjects. JAMA (2013) 310:2191-4. doi:10.1001/jama.2013.281053

30. Wittchen HU, Zaudig M, Fydrich T. Strukturiertes Klinisches Interview für DSM-IV. Göttingen: Hogrefe Verlag (1997).

31. American Psychiatric Association. Diagnostic and Statistical Manual of Mental Disorders, Text Revision (DSM-IV-TR). 4th ed. Washington, DC: American Psychiatric Publishing (2000).

32. Beck AT, Steer RA, Ball R, Ranieri W. Comparison of beck depression inventories IA and -II in psychiatric outpatients. JPers Assess (1996) 67:588-97. doi:10.1207/s15327752jpa6703_13

33. Schellig D, Schächtele B. Visueller \& Verbaler Merkfähigkeitstest. Frankfurt am Main: Pearson Assessment \& Information (2001).

34. Härting C, Markowitsch HJ, Neufeld H, Calabrese P, Deisinger K, Kessler J. WMS-R - Wechsler Gedächtnistest-revidierte Fassung. Bern: Verlag Hans Huber (2000).

35. Nasreddine ZS, Phillips NA, Bédirian V, Charbonneau S, Whitehead V, Collin I, et al. The Montreal Cognitive Assessment, MoCA: a brief screening tool for mild cognitive impairment. J Am Geriatr Soc (2005) 53:695-9. doi:10.1111/j.1532-5415.2005.53221.x

36. Costa AS, Reich A, Fimm B, Ketteler ST, Schulz JB, Reetz K. Evidence of the sensitivity of the MoCA alternate forms in monitoring cognitive change in early Alzheimer's disease. Dement Geriatr Cogn Disord (2014) 37:95-103. doi:10.1159/000351864

37. Della Sala S, Gray C, Baddeley A, Allamano N, Wilson L. Pattern span: a tool for unwelding visuo-spatial memory. Neuropsychologia (1999) 37:1189-99. doi:10.1016/S0028-3932(98)00159-6

38. Lehrl S. Mehrfachwahl-Wortschatz-Intelligenztest MWT-B. 5th ed. Balingen: Spitta Verlag (2005). 
39. Peirce JW. PsychoPy-psychophysics software in python. J Neurosci Methods (2007) 162:8-13. doi:10.1016/j.jneumeth.2006.11.017

40. R Core Team. R: A Language and Environment for Statistical Computing. Vienna: R Foundation for Statistical Computing (2016).

41. Xu R. Measuring explained variation in linear mixed effects models. Stat Med (2003) 22:3527-41. doi:10.1002/sim.1572

42. Ward BD. Simultaneous Inference for fMRI Data. AFNI 3dDeconvolve Documentation. Milwaukee, WI: Medical College of Wisconsin (2000).

43. Olejnik S, Algina J. Generalized eta and omega squared statistics: measures of effect size for some common research designs. Psychol Methods (2003) 8: 434-47. doi:10.1037/1082-989x.8.4.434

44. Seth AK, Barrett AB, Barnett L. Granger causality analysis in neuroscience and neuroimaging. J Neurosci (2015) 35:3293-7. doi:10.1523/JNEUROSCI. 4399-14.2015

45. Kurth F, Luders E, Gaser C. VBM8 Toolbox Manual. (2010).

46. Hayasaka S, Phan KL, Liberzon I, Worsley KJ, Nichols TE. Nonstationary cluster-size inference with random field and permutation methods. Neuroimage (2004) 22:676-87. doi:10.1016/j.neuroimage.2004.01.041

47. Oostra KM, Oomen A, Vanderstraeten G, Vingerhoets G. Influence of motor imagery training on gait rehabilitation in sub-acute stroke: a randomized controlled trial. J Rehabil Med (2015) 47:204-209. doi:10.2340/165019771908

48. Murphy SE, Clare O'Donoghue M, Drazich EHS, Blackwell SE, Christina Nobre A, Holmes EA. Imagining a brighter future: the effect of positive imagery training on mood, prospective mental imagery and emotional bias in older adults. Psychiatry Res (2015) 230:36-43. doi:10.1016/j.psychres. 2015.07.059

49. Papadelis C, Kourtidou-Papadeli C, Bamidis P, Albani M. Effects of imagery training on cognitive performance and use of physiological measures as an assessment tool of mental effort. Brain Cogn (2007) 64:74-85. doi:10.1016/j. bandc.2007.01.001

50. Nellessen N, Rottschy C, Eickhoff S, Ketteler S, Kuhn H, Shah NJ, et al. Specific and disease stage-dependent episodic memory-related brain activation patterns in Alzheimer's disease: a coordinate-based meta-analysis. Brain Struct Funct (2014) 220:1-17. doi:10.1007/s00429-014-0744-6

51. Miotto EC, Savage CR, Evans JJ, Wilson BA, Martins MG, Iaki S, et al. Bilateral activation of the prefrontal cortex after strategic semantic cognitive training. Hum Brain Mapp (2006) 27:288-95. doi:10.1002/hbm.20184

52. Brehmer Y, Rieckmann A, Bellander M, Westerberg H, Fischer H, Bäckman L. Neural correlates of training-related working-memory gains in old age. Neuroimage (2011) 58:1110-20. doi:10.1016/j.neuroimage.2011.06.079

53. Bueichekú E, Miró-Padilla A, Palomar-García M-Á, Ventura-Campos N, Parcet M-A, Barrós-Loscertales A, et al. Reduced posterior parietal cortex activation after training on a visual search task. Neuroimage (2016) 135:204-13. doi:10.1016/j.neuroimage.2016.04.059

54. Zotev V, Yuan H, Misaki M, Phillips R, Young KD, Feldner MT, et al. Correlation between amygdala BOLD activity and frontal EEG asymmetry during real-time fMRI neurofeedback training in patients with depression. Neuroimage Clin (2016) 11:224-38. doi:10.1016/j.nicl.2016.02.003

55. Ranganath C, D’Esposito M. Medial temporal lobe activity associated with active maintenance of novel information. Neuron (2001) 31:865-73. doi:10.1016/S0896-6273(01)00411-1

56. Stern CE, Sherman SJ, Kirchhoff BA, Hasselmo ME. Medial temporal and prefrontal contributions to working memory tasks with novel and familiar stimuli. Hippocampus (2001) 11:337-46. doi:10.1002/hipo.1048

57. Hasselmo ME, Stern CE. Mechanisms underlying working memory for novel information. Trends Cogn Sci (2006) 10:487-93. doi:10.1016/j.tics.2006. 09.005

58. Nee DE, Brown JW, Askren MK, Berman MG, Demiralp E, Krawitz A, et al. Meta-analysis of executive components of working memory. Cereb Cortex (2013) 23:264-82. doi:10.1093/cercor/bhs007

59. Rottschy C, Langner R, Dogan I, Reetz K, Laird AR, Schulz JB, et al. Modelling neural correlates of working memory: a coordinate-based meta-analysis. Neuroimage (2012) 60:830-46. doi:10.1016/j.neuroimage.2011.11.050

60. Bartha L, Brenneis C, Schocke M, Trinka E, Köylü B, Trieb T, et al. Medial temporal lobe activation during semantic language processing: fMRI findings in healthy left- and right-handers. Brain Res Cogn Brain Res (2003) 17:339-46. doi:10.1016/S0926-6410(03)00135-6
61. Noack H, Lövdén M, Schmiedek F. On the validity and generality of transfer effects in cognitive training research. Psychol Res (2014) 78:773-89. doi:10.1007/s00426-014-0564-6

62. Robins A. Transfer in cognition. Conn Sci (1996) 8:185-204. doi:10.1080/ 095400996116875

63. Matzen LE, Trumbo MC, Haass MJ, Hunter MA, Silva A, Stevens-Adams SM, et al. Practice makes imperfect: working memory training can harm recognition memory performance. Mem Cognit (2016) 44:1-15. doi:10.3758/ s13421-016-0629-4

64. Sperling R. The potential of functional MRI as a biomarker in early Alzheimer's disease. Neurobiol Aging (2011) 32:S37-43. doi:10.1016/j. neurobiolaging.2011.09.009

65. Cavanna AE, Trimble MR. The precuneus: a review of its functional anatomy and behavioural correlates. Brain (2006) 129:564-83. doi:10.1093/brain/awl004

66. Wagner AD, Shannon BJ, Kahn I, Buckner RL. Parietal lobe contributions to episodic memory retrieval. Trends Cogn Sci (2005) 9:445-53. doi:10.1016/j. tics.2005.07.001

67. Bruner E, Jacobs H. Alzheimer's disease: the downside of a highly evolved parietal lobe? J Alzheimers Dis (2013) 35:227-40. doi:10.3233/JAD-122299

68. Dickerson BC, Sperling RA. Functional abnormalities of the medial temporal lobe memory system in mild cognitive impairment and Alzheimer's disease: insights from functional MRI studies. Neuropsychologia (2008) 46:1624-35. doi:10.1016/j.neuropsychologia.2007.11.030

69. Rizio AA, Dennis NA. The neural correlates of cognitive control: successful remembering and intentional forgetting. J Cogn Neurosci (2012) 25:1-16. doi:10.1162/jocn_a_00310

70. Ridderinkhof KR, Ullsperger M, Crone EA, Nieuwenhuis S. The role of the medial frontal cortex in cognitive control. Science (2004) 306:443-7. doi:10.1126/science.1100301

71. D'Esposito M, Aguirre G, Zarahn E, Ballard D, Shin R, Lease J. Functional MRI studies of spatial and nonspatial working memory. Brain Res Cogn Brain Res (1998) 7:1-13. doi:10.1016/S0926-6410(98)00004-4

72. Takeuchi H, Taki Y, Nouchi R, Hashizume H, Sekiguchi A, Kotozaki Y, et al. Effects of multitasking-training on gray matter structure and resting state neural mechanisms. Hum Brain Mapp (2014) 35:3646-60. doi:10.1002/ hbm. 22427

73. Zhang K, Wang H, Dong G, Wang M, Zhang J, Zhang H, et al. Neural activation during imitation with or without performance feedback: an fMRI study. Neurosci Lett (2016) 629:202-7. doi:10.1016/j.neulet.2016.07.015

74. Sailer U, Robinson S, Fischmeister FPS, Moser E, Kryspin-Exner I, Bauer H. Imaging the changing role of feedback during learning in decision-making. Neuroimage (2007) 37:1474-86. doi:10.1016/j.neuroimage.2007.07.012

75. Seth AK, Chorley P, Barnett LC. Granger causality analysis of fMRI BOLD signals is invariant to hemodynamic convolution but not downsampling. Neuroimage (2013) 65:540-55. doi:10.1016/j.neuroimage.2012.09.049

76. Friston K, Moran R, Seth AK. Analysing connectivity with Granger causality and dynamic causal modelling. Curr Opin Neurobiol (2013) 23:172-8. doi:10.1016/j.conb.2012.11.010

77. Wen X, Rangarajan G, Ding M. Is Granger causality a viable technique for analyzing fMRI data? PLoS One (2013) 8:e67428. doi:10.1371/journal. pone. 0067428

78. Deshpande G, Hu X. Investigating effective brain connectivity from fMRI data: past findings and current issues with reference to Granger causality analysis. Brain Connect (2012) 2:235-45. doi:10.1089/brain.2012.0091

79. Sulzer J, Haller S, Scharnowski F, Weiskopf N, Birbaumer N, Blefari ML, et al. Real-time fMRI neurofeedback: progress and challenges. Neuroimage (2013) 76:386-99. doi:10.1016/j.neuroimage.2013.03.033

80. Jaeggi SM, Buschkuehl M, Shah P, Jonides J. The role of individual differences in cognitive training and transfer. Mem Cognit (2014) 42:464-80. doi:10.3758/ s13421-013-0364-z

81. Liberati G, Dalboni da Rocha JLL, van der Heiden L, Raffone A, Birbaumer N, Olivetti Belardinelli $\mathrm{M}$, et al. Toward a brain-computer interface for Alzheimer's disease patients by combining classical conditioning and brain state classification. J Alzheimers Dis (2012) 31(Suppl 3):S211-20. doi:10.3233/ jad-2012-112129

82. Gani C, Birbaumer N, Strehl U. Long term effects after feedback of slow cortical potentials and of theta-beta-amplitudes in children with attention-deficit/ hyperactivity disorder (ADHD). Int J Bioelectromagn (2008) 10:209-32. 
83. Zhuo C, Li L. The application and efficacy of combined neurofeedback therapy and imagery training in adolescents with Tourette syndrome. J Child Neurol (2013) 29:965-8. doi:10.1177/0883073813479999

84. Steiner NJ, Frenette EC, Rene KM, Brennan RT, Perrin EC. In-school neurofeedback training for ADHD: sustained improvements from a randomized control trial. Pediatrics (2014) 133:483-92. doi:10.1542/peds.20132059

85. Strehl U, Birkle SM, Wörz S, Kotchoubey B. Sustained reduction of seizures in patients with intractable epilepsy after self-regulation training of slow cortical potentials - 10 years after. Front Hum Neurosci (2014) 8:1-7. doi:10.3389/ fnhum.2014.00604

86. Yoo S-S, Lee J-H, O’Leary H, Panych LP, Jolesz FA. Neurofeedback fMRImediated learning and consolidation of regional brain activation during motor imagery. Int J Imaging Syst Technol (2008) 18:69-78. doi:10.1002/ ima.20139
87. Zilverstand A, Sorger B, Sarkheil P, Goebel R. fMRI neurofeedback facilitates anxiety regulation in females with spider phobia. Front Behav Neurosci (2015) 9:148. doi:10.3389/fnbeh.2015.00148

Conflict of Interest Statement: The authors declare that the research was conducted in the absence of any commercial or financial relationships that could be construed as a potential conflict of interest.

Copyright () 2017 Hohenfeld, Nellessen, Dogan, Kuhn, Müller, Papa, Ketteler, Goebel, Heinecke, Shah, Schulz, Reske and Reetz. This is an open-access article distributed under the terms of the Creative Commons Attribution License (CC BY). The use, distribution or reproduction in other forums is permitted, provided the original author(s) or licensor are credited and that the original publication in this journal is cited, in accordance with accepted academic practice. No use, distribution or reproduction is permitted which does not comply with these terms. 